OPEN ACCESS

Edited by:

Mikhail Zheludkevich, Helmholtz-Zentrum Geesthacht, Germany

Reviewed by: Wolfram Fürbeth, DECHEMA-Forschungsinstitut, Germany

loannis A. Kartsonakis, National Technical University of Athens, Greece

${ }^{*}$ Correspondence: Alexander Lutz alexander.lutz@vub.ac.be

Specialty section: This article was submitted to

Corrosion Research,

a section of the journal

Frontiers in Materials

Received: 24 July 2015 Accepted: 07 December 2015 Published: 07 January 2016

Citation: Lutz A, van den Berg O, Wielant J, De Graeve I and Terryn H (2016) A Multiple-Action Self-Healing Coating. Front. Mater. 2:73 doi: 10.3389/fmats.2015.00073

\section{A Multiple-Action Self-Healing Coating}

\author{
Alexander Lutz ${ }^{1,2 *}$, Otto van den Berg ${ }^{3}$, Jan Wielant ${ }^{4}$, Iris De Graeve ${ }^{1}$ and Herman Terryn ${ }^{1}$ \\ ${ }^{1}$ Research Group Electrochemical and Surface Engineering, Department of Materials and Chemistry, Vrije Universiteit \\ Brussel, Elsene, Belgium, ${ }^{2}$ SIM vZw, Zwijnaarde, Belgium, ${ }^{3}$ Polymer Chemistry Research Group, Department of Organic and \\ Macromolecular Chemistry, Ghent University, Ghent, Belgium, ${ }^{4}$ OCAS NV, Zelzate, Belgium
}

This article describes a self-healing coating for corrosion protection of metals, which combines two different types of self-healing mechanisms in one coating with multiple-healing functionality. 2-Mercaptobenzothiazole (MBT) was loaded into layered double hydroxide $(\mathrm{LDH})$ carriers that were mixed into an acrylated polycaprolactone polyurethane-based shape-recovery coating and applied on hot-dip galvanized steel (HDG). The effect of triggered release of MBT on the protection of HDG became visible when samples with manually applied defects in the coating were immersed in $0.05 \mathrm{M} \mathrm{NaCl}$ solution (first, autonomous-healing mechanism). The shape recovery (second, non-autonomous-healing mechanism) was triggered by heating the samples for 2 min to $60^{\circ} \mathrm{C}$. SEM-EDX and Raman spectroscopy proved the presence of MBT in the LDH, in the MBT-loaded LDH in the coating and the released MBT on the HDG surface in the damaged area after being in contact with a solution containing corrosive ions. Electrochemical impedance spectroscopy and scanning vibrating electrode technique demonstrate the corrosion protection effect of MBT in the coating with a defect and the restoration of the barrier properties of the coating after defect closure. This way, the independent mechanisms of this multi-action self-healing coating could be demonstrated.

Keywords: self-healing, EIS, Raman spectroscopy, polymer coatings, SVET, corrosion inhibitor

\section{INTRODUCTION}

Many researchers are trying to tackle the immanent problem of corrosion by using self-healing coatings for all kinds of applications (off-shore wind parks, airplanes, automotive industry, chromium replacement, etc.). Their work led to different sorts of self-healing mechanisms being developed, which fulfill different requirements. Several reviews exist which discuss the latest advances in the field (Zwaag, 2007; Wu et al., 2008; Yuan, 2008; Blaiszik et al., 2010; Samadzadeh et al., 2010; Fedrizzi, 2011; García et al., 2011; Zadeh et al., 2013). There are coatings that physically close or fill defects (Toohey et al., 2007; Jorcin et al., 2010; González-García et al., 2011a,b; Brancart et al., 2012), others that automatically protect the metal substrate underneath the coating against corrosion induced by aggressive species (Andreeva and Shchukin, 2008; Shchukin et al., 2010; Zheludkevich et al., 2010; Yasakau et al., 2012; Borisova et al., 2013a,b). Alternatively, self-healing coatings can also be split in two categories: (i) intrinsically healing coatings where the coating matrix performs the healing (Rodriguez et al., 2011; Döhler et al., 2015; Lutz et al., 2015) and (ii) extrinsically healing coatings that embody containers within the coating matrix that release a healing agent (Toohey et al., 2007; Hansen et al., 2009; Zheludkevich et al., 2010; Maia et al., 2012; Borisova et al., 2013b). Some coating systems are thus fully autonomous (Brown et al., 2003; Maia et al., 2012, 2013; Hillewaere and Du 
Prez, 2015) and their triggers are either species that are generated by the corrosion process (Grigoriev et al., 2009; Zheludkevich et al., 2012) or the infliction of a defect (White et al., 2001; Brown et al., 2003; Toohey et al., 2007; Hansen et al., 2009; Hillewaere and Du Prez, 2015). Other coatings, called non-autonomous or on-demand healing systems, require a trigger, such as heat to initiate the healing process (D'Hollander et al., 2009; Wouters et al., 2009; Jorcin et al., 2010; González-García et al., 2011a,b; Brancart et al., 2012; Luo and Mather, 2013; Scheltjens et al., 2013; Lutz et al., 2015).

As reviewed by García et al. (2011), each self-healing mechanism not only has its advantages but also has its disadvantages. In the last 2 years, the first combinations of healing agents have been presented (Hong et al., 2007; Markley et al., 2007; Montemor et al., 2008, 2012; Rodriguez et al., 2011; Ferrer et al., 2014; Kartsonakis et al., 2014; Serdechnova et al., 2014; Döhler et al., 2015). Yet, the focus of these developments has been set on extending one sort of healing mechanism [e.g., better corrosion inhibition by using a second corrosion inhibitor (Hong et al., 2007; Markley et al., 2007; Montemor et al., 2008, 2012; Ferrer et al., 2014; Kartsonakis et al., 2014; Serdechnova et al., 2014) or achieving better scratch closing by adding another polymer that links the two surface boundaries of a closed scratch (Rodriguez et al., 2011; Döhler et al., 2015)]. It does, however, not solve the basic issues of single action healing systems in the former case, the scratch will stay open for continuous access of corrosive species and in the latter case the surface in the defect is not protected until after the scratch closing is triggered.

Therefore, we developed a multi-action self-healing (MASH) coating that combines an extrinsic autonomous corrosion protection system with an intrinsic on-demand shape-recovery coating. To underline the importance of the combination of the two systems, it is necessary to understand the limitations of the single self-healing systems; these ones being just examples for others: the corrosion inhibitor system alone would after a (long enough) period of time be depleted and no longer be able to locally release corrosion inhibitors. The absence of certain species still enables corrosion but does not trigger the release of the inhibitor. Also, the visual appearance of a product with scratches is no longer flawless, and thus loses its esthetical value. Generally, such autonomous systems protect the metal substrate, but lack the ability to restore the coatings' initial barrier properties. The release of an actively self-healing agent (corrosion inhibitor, water repellent, coating repair agent, etc.) reduces the barrier functionality at the original location of the container where it was stored in trade for the corrosion inhibition in the defect. Additionally, the amount of locally stored self-healing agent is small compared to the to-be-healed defect size. On the other hand, the shaperecovery coating does not provide any protection if the healing is not triggered. Consequently, delamination and corrosion in the defect and under the coating can hinder the scratch closing process. The combination of the two parallel healing systems tackles these disadvantages. The corrosion inhibitor can reduce delamination of a not yet healed defect and when the scratch is closed, further corrosion inhibitor depletion is prevented.

Scheme 1 shows the working principle of this MASH coating system: the self-healing polymer coating contains finely dispersed corrosion inhibitor containers (Scheme 1A). For this study, 2-mercaptobenzothiazole (MBT) was chosen as a corrosion inhibitor (Müller and Imblo, 1996; Bastos et al., 2008) and as shown by Tedim et al. intercalated in between the octahedral layers of layered double hydroxides (LDHs) (Poznyak et al., 2009). Because LDH can be loaded with a lot of different kinds of negatively charged ions, it readily releases the MBT anions at elevated $\mathrm{pH}$ from the crystal lattice in exchange for smaller and highly charged chloride ions (Kukkadapu et al., 1997; He et al., 2006; Poznyak et al., 2009). This inhibitor release mechanism works fully autonomous as soon as the $\mathrm{pH}$ is high enough and/or chloride ions are present in the solution. Such nano- to micrometer sized particles were embedded into a shape-recovery coating based on polycaprolactone and isobornylacrylate (Lutz et al., 2015). The combination of the two blocks results in a high molecular weight PCL acrylate with good mechanical properties with shape-recovery functionality above the glass transition temperature of the PCL phase. Heating this coating to $60^{\circ} \mathrm{C}$ induces enough mobility to automatically close mechanically inflicted defects, such as scratches or indentations (Scheme 1B) within only $2 \mathrm{~min}$. This mechanism is fully independent of the MBT-release mechanism as sketched in Scheme 1C. However, if the scratch in the coating is not immediately healed, water and corrosive species can reach the metal and initiate corrosion. In this case, the corrosion inhibitor containers close to the scratch locally release the MBT into the solution and take up chloride ions in exchange. The released MBT dissolves and diffuses to the metal surface to form a protection layer on top of it (Scheme 1D). The shape-recovery mechanism can still be triggered independently of the first mechanism and additionally close the scratch at a later moment (Scheme 1E).

In this study, the structure and composition of the MASH coating was analyzed by SEM-EDX and Raman spectroscopy to investigate the inorganic (LDH and metal surface) and organic parts (MBT and coating) of this composite material, respectively. The effect of the released corrosion inhibitor and the independent restoration of the barrier properties of the coating after heating were evaluated by electrochemical impedance spectroscopy (EIS), whereas scanning vibrating electrode technique (SVET) was used to indicate the different corrosion activity of defects in a coating. Finally, the delaminated areas underneath the coatings after immersion were compared under an optical microscope and the presence of the corrosion inhibitor on the metal surface in the case of the MASH sample was confirmed by Raman spectroscopy.

\section{EXPERIMENTAL}

\section{Synthesis}

The shape-recovery-based self-healing polymer coating resin was prepared as described in a recent article (Lutz et al., 2015). In short, the prepolymer - a telechelic acrylated polycaprolactone (36 mol\% PCL in the final formulation) - was mixed with isobornylacrylate at $60^{\circ} \mathrm{C}$. 2,2-Dimethoxy-2-phenylacetophenone (10 mg/g, 99\%, from Sigma-Aldrich Co.) and KIP 160 (a difunctional alpha hydroxy ketone, $10 \mathrm{mg} / \mathrm{g}$ from Allnex Belgium NV) were added to the mixture as photoinitiators. This resin was applied by a bar coater, resulting in a dry film thickness of about $100 \mu \mathrm{m}$ on hot-dip galvanized steel (HDG) 


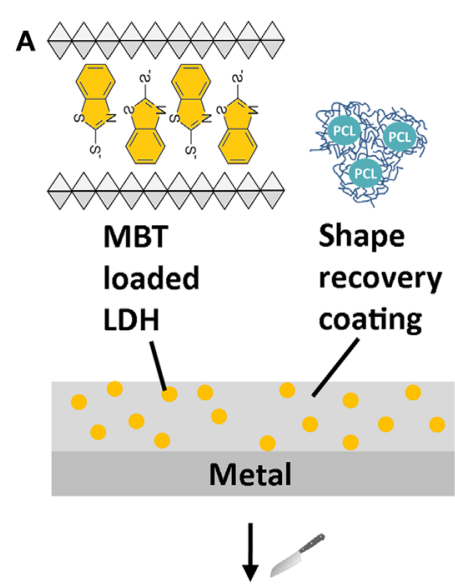

B
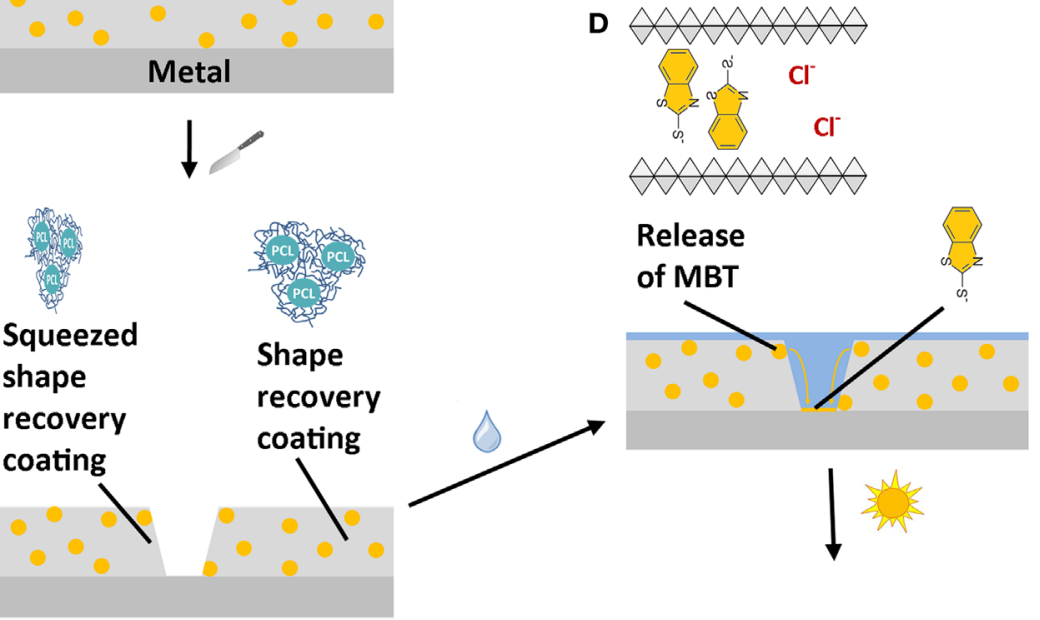

E

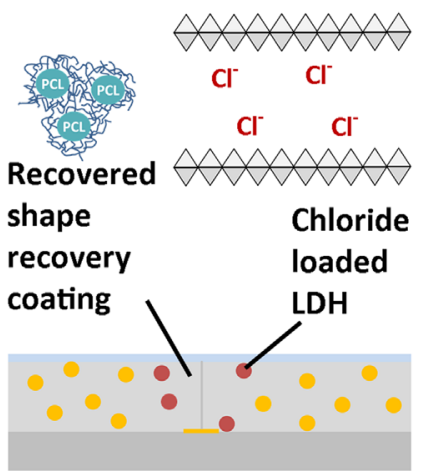

SCHEME 1 | Working mechanism of a multi-action self-healing (MASH) coating (A): when a MASH coating is scratched (B) two independent actions can follow: (1) the scratch is closed by heating the sample and restoring the coating barrier (C). (2) If a solution containing, for example, aggressive chloride ions reaches the defect, the corrosion inhibitor MBT is released from LDH in exchange for the chloride ions. The released MBT forms a protective layer protecting the metal substrate (D). Eventually, the scratch closing action by heating can still be triggered to prevent continuous access of aggressive ions (E).

sheets (S250GD + Z350 from ArcelorMittal; thickness $0.8 \mathrm{~mm}$ ) and cured by passing twice under $210 \mathrm{~W} / \mathrm{cm}$ UV lamps $(\mathrm{H}+\mathrm{D})$ under nitrogen atmosphere. Before coating application, the steel samples were cleaned in two steps: (i) vapor cleaning in Kerry Ultrasonics installation and (ii) alkaline degreasing with Gardoclean S5086. The samples were prepared without the use of any other corrosion protection pretreatment of the HDG steel or an adhesion promoter to avoid unambiguous results and have clearly visible coating delamination. This sample is the reference and will be called $\mathrm{SH}$.

Layered double hydroxides were synthesized following a procedure similar to the one of Poznyak et al. (2009). All solutions were prepared with boiled distilled water minimizing the amount of dissolved $\mathrm{CO}_{2}$ in the solutions. Approximately $0.5 \mathrm{M} \mathrm{Zn}\left(\mathrm{NO}_{3}\right)_{2} \times 6 \mathrm{H}_{2} \mathrm{O}$ and $0.25 \mathrm{M} \mathrm{Al}\left(\mathrm{NO}_{3}\right)_{3} \times 9 \mathrm{H}_{2} \mathrm{O}$ were added dropwise to a $1.5 \mathrm{M} \mathrm{NaNO}_{3}$ solution at $\mathrm{pH} 10$ and kept constant $( \pm 1)$ at this $\mathrm{pH}$ by adding simultaneously drops of $2 \mathrm{M} \mathrm{NaOH}$. Nitrogen was bubbled through the reaction volume to avoid $\mathrm{CO}_{2}$ contamination. Afterwards the closed glass bottle with the solution was put into an oven at $65^{\circ} \mathrm{C}$ for $24 \mathrm{~h}$. Subsequently, the white suspension was centrifuged and washed with boiled distilled water several times. The anion-exchange reaction was performed by adding the LDH slurry to a saturated solution of 2-MBT under argon atmosphere and stirring the closed bottle 


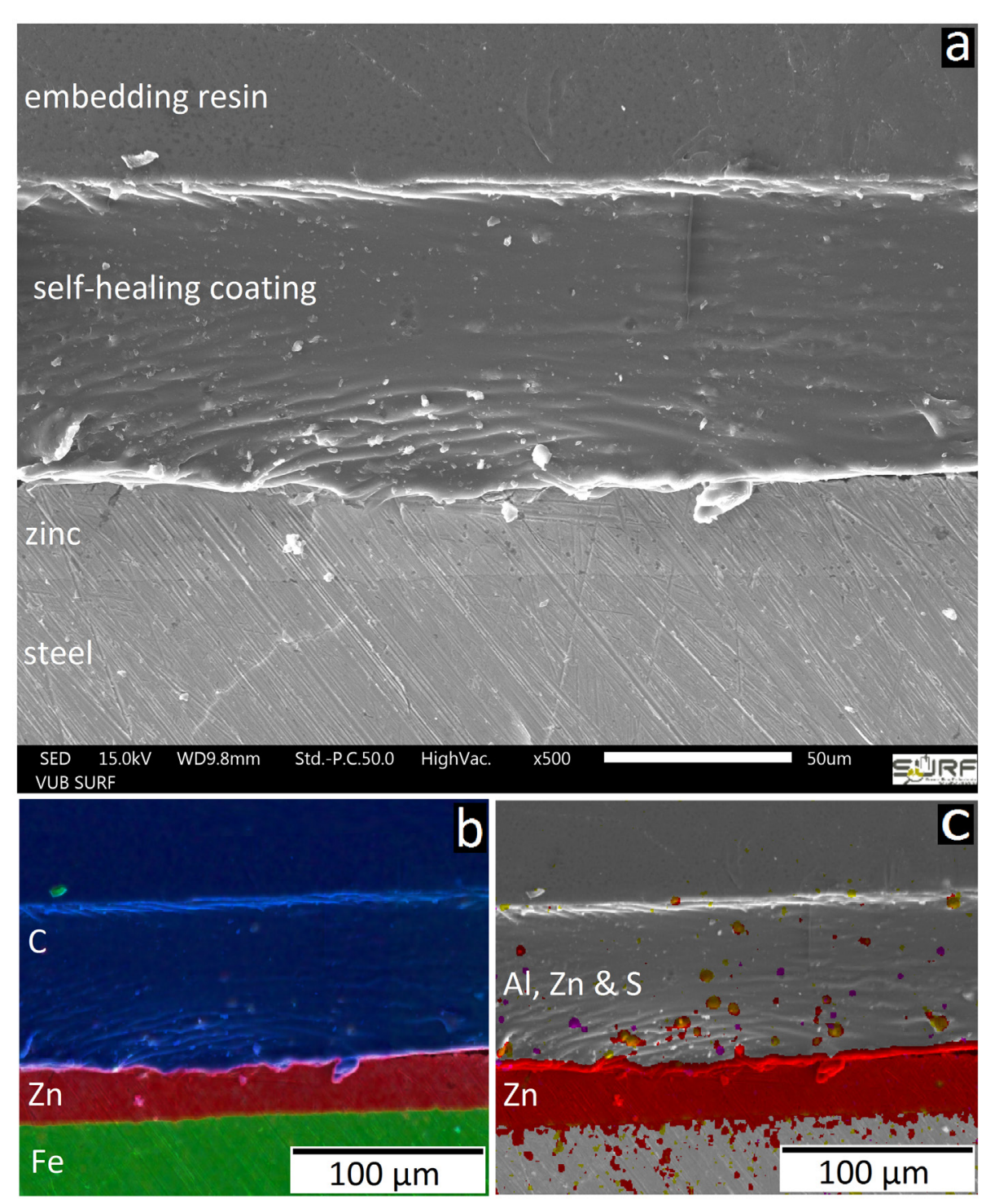

FIGURE 1 | The SEM image (A) shows the structure of the MASH sample in cross-section. EDX mappings show the different layers (B) and the finely distributed corrosion inhibitor containers (C)

overnight. The solution was centrifuged and the anion-exchange reaction was repeated a second time. The slurry was washed with boiled distilled water and centrifuged twice again and dried in an oven at $65^{\circ} \mathrm{C}$. The dry MBT-loaded LDH (MLDH) was grinded to a fine powder with a mortar and pestle, mixed into the $\mathrm{SH}$ coating resin at $60^{\circ} \mathrm{C}$ and put into the sonication bath for $10 \mathrm{~min}$. Subsequently, the MASH resin was applied on HDG the same way as the reference $(\mathrm{SH})$ coating with a $100 \mu \mathrm{m}$ bar coater and cured with $210 \mathrm{~W} / \mathrm{cm}$ UV lamps under nitrogen atmosphere. This sample is called MASH.

\section{Analysis}

Electrochemical impedance spectroscopy was performed using an Autolab 128N potentiostat from Metrohm AG (Swiss) and a three-electrode setup (the sample as working electrode, a platinum grid as counter electrode, and a $\mathrm{Ag} \mid \mathrm{AgCl}$ reference electrode coupled with a Pt wire and a capacitor as a high-frequency bypass). Forty-nine frequencies were excited, logarithmically equally distributed over the whole measurement range from $10^{5}$ to $10^{-1} \mathrm{~Hz}$ with an amplitude of $10 \mathrm{mV} \mathrm{rms}$. A $1 \mathrm{~cm}^{2}$ surface of the samples was exposed to a $0.05 \mathrm{M}$ sodium chloride $(\mathrm{NaCl})$ solution and was left immersed for about 5 min until a stable open circuit potential (OCP) was reached. All defects within this work were inflicted with a handmade microchisel that was pushed perpendicularly to the surface into the immersed coating. The size of the formed defect was roughly 50 by $500 \mu \mathrm{m}$. The microchisel was manufactured by sharpening a screwdriver with sand paper of 500, 1200, and, finally, 4000 grit to create a knifelike edge.

Scanning vibrating electrode technique measurements were performed using a SVET from Applicable Electronics, LLC, in 
combination with the ASET software from Science Wares Inc. (USA). For every $35 \mu \mathrm{m}$, one measurement point of the vertical component of the current density was recorded to build up the entire current density map. For SVET, the coated HDG samples were covered with adhesive tape (3M polyester tape No. 8402) except for the measurement area $\left(\sim 2 \mathrm{~mm}^{2}\right)$. Within this area, two defects were made as described above (see Figure 3). Full penetration of the organic coating, exposing the underlying HDG steel, was confirmed under an optical microscope. The samples were put on a hot-stage and heated to $60^{\circ} \mathrm{C}$ for $2 \mathrm{~min}$ and then put on a lab table to cool down to room temperature.

Optical microscopy was performed on a Leica stereomicroscope together with its official LAX software. The SEM images were acquired with JSM-IT300 from Jeol (Europe) BV at 10 and $15 \mathrm{kV}$. The instrument was coupled with an Oxford Instruments SDD X-MaxN $80 \mathrm{~mm}^{2}$ EDS detector for elemental analysis. Raman spectroscopy was performed with a LabRAM HR Evolution confocal Raman spectroscope from Horiba Scientific. A $532 \mathrm{~nm}$ wavelength green laser, a 50× long distance objective, a $600 \mathrm{~g} /$ mm grating, and an air cooled CCD detector were used to record the Raman spectra. These data were processed with the standard LabSpec 6.2 software for this instrument, including spike removal and background correction on each single spectrum.

The experiments were performed at least three times, and similar results were achieved each time. For better comparison, the measurements shown in the results are from a single, representative sample. The coating was manually peeled off with a scalpel knife for the Raman spectroscopic measurements on the HDG surface after $48 \mathrm{~h}$ immersion in $0.05 \mathrm{M} \mathrm{NaCl}$ solution.

\section{RESULTS AND DISCUSSION}

\section{Coating Characterization}

The SEM cross-section in Figure 1 shows a cross-section of the MASH sample. Figure 1A presents the layers of steel and zinc

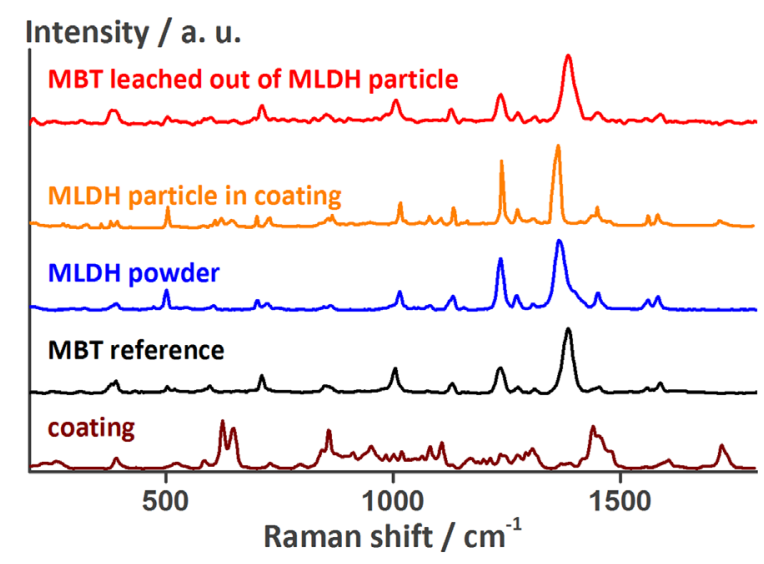

FIGURE 2 | Raman spectra of a MLDH particle in the MASH coating, of the non-embedded MLDH powder, of MBT, and of the self-healing coating next to the MLDH particle confirming the presence of MBT encapsulated in the MASH coating. The peak assignment can be found in the Table S1 in the Supplementary Material. from the substrate and the self-healing coating on top. The elemental mapping (Figure 1B) confirms this structure. The MASH sample also contains finely dispersed $\mathrm{LDH}$ particles in the coating which contain the corrosion inhibitor MBT. These micrometer sized particles can also be seen as brighter dots in the self-healing coating in Figure 1A. Again, the EDX mapping (Figure 1C) confirms the elements $\mathrm{Al}$ and $\mathrm{Zn}$ (from $\mathrm{LDH}$ ) and $\mathrm{S}$ (from MBT), all present at the same locations.

A Raman spectrum of one of these MLDH particles in the coating is presented in Figure 2. The main peaks of this spectrum colored in orange match the ones of the Raman spectrum of loose LDH particles loaded with MBT (blue). Figure 2 also shows a reference spectrum (black) of a drop of MBT solution put on
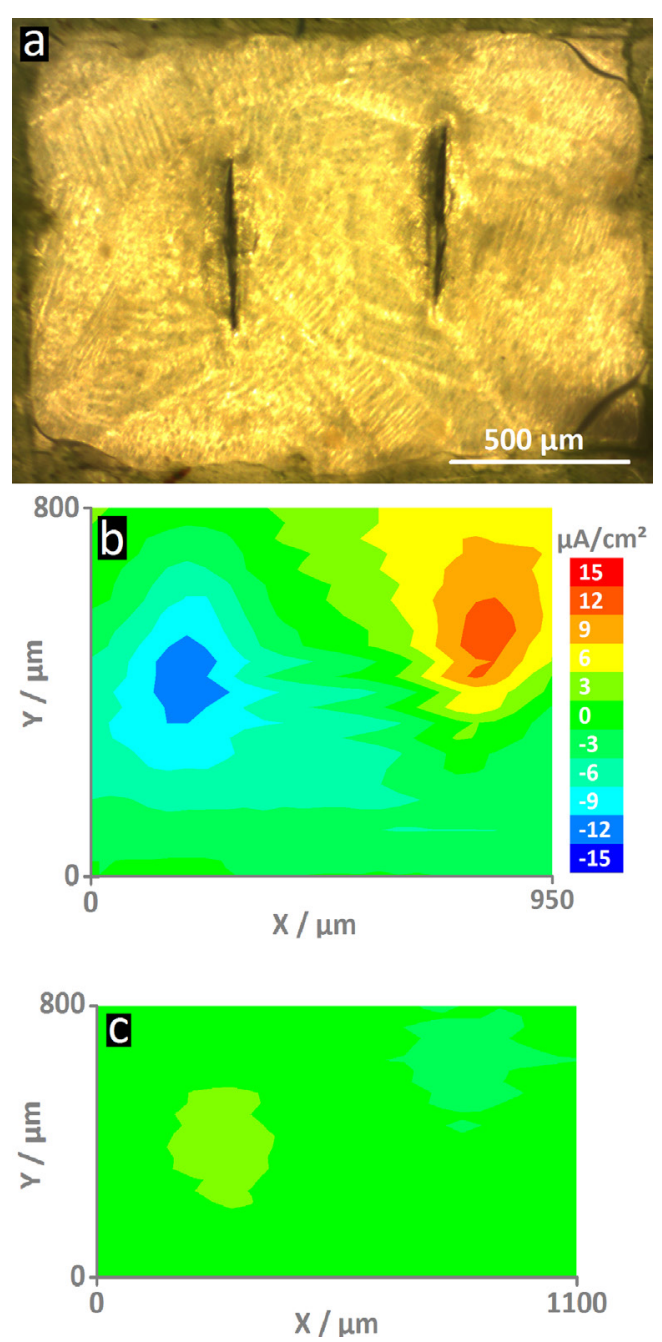

FIGURE 3 | The optical micrograph (A) shows the MASH sample surface (The reference sample looks similar.). In the center are two vertical screwdriver blade indentations that form the defects through the coating. The ionic current density maps were recorded after $4 \mathrm{~h}$ of immersion $100 \mu \mathrm{m}$ on top of the surfaces. For comparison, both maps have the same scale. The map of the reference sample (B) shows high current densities $\left( \pm 12 \mu \mathrm{A} / \mathrm{cm}^{2}\right)$ - an indication for the actively corroding sample. On the contrary, the sample with MLDH (C) shows much smaller current densities and thus corrosion inhibition. 
an in-house developed SERS probe containing finely dispersed silver nanoparticles. The peaks are slightly shifted because of the different substrate, but coincide with those reported in the literature (Yang et al., 2008). An aqueous solution of MLDH powder was also put directly on HDG and the Ag-SERS probe. In both cases no MBT could be detected. This is not surprising, because in near-neutral solution the ion-exchange mechanism to release MBT is not triggered. On the contrary, when a drop of solution containing $\mathrm{MLDH}$ particles together with $\mathrm{Na}_{2} \mathrm{CO}_{3}$ was put on the Ag-SERS probe, the $\mathrm{pH}$ of the solution was high enough to cause the release of MBT. In this case, the red spectrum (top) in Figure 2 was recorded. It also shows that the MBT molecule first encapsulated in $\mathrm{LDH}$ can be released again unaltered into the solution. Finally, the Raman spectrum of MLDH particles in the coating contains weak peaks of the surrounding coating. Because of the MLDH particle size and the similar laser spot size, it is not surprising that the information gathered with the Raman spectrometer contains information of the material surrounding the MLDH particle. These measurements confirm the successful embedding of the corrosion inhibitor MBT encapsulated in $\mathrm{LDH}$ in the self-healing coating.

\section{First Self-Healing Action: Effect of MBT}

The restoration of the barrier properties of the self-healing coating alone were already demonstrated earlier (Lutz et al., 2015). Therefore, in this publication, the focus is set on the additional corrosion protection of released MBT before thermal healing of the coating is induced. The corrosion inhibition effect was investigated using SVET and EIS.

Figure 3A shows the area of the MASH coating with two defects as measured by SVET. Because the coating is transparent, the typical HDG steel surface can still vaguely be seen. The green insulation tape - visible at the outside borders of the image - ensures that all actively corroding areas will be recorded by the vibrating probe. The corrosion activity was measured as ion flux above defects in the reference coating and the MASH coating by SVET $4 \mathrm{~h}$ after immersion and $100 \mu \mathrm{m}$ above the surface. Figure 3B shows the high current densities (of cathodic and anodic currents) up to about $\pm 12 \mu \mathrm{A} / \mathrm{cm}^{2}$ on the map above the artificial defects (or scribes) in the reference coating. Because no corrosion inhibitors were added, corrosive attack took place in the defects where the metal is exposed to the aggressive solution. The maximum current densities in the SVET map just above the defects in the MASH coating are much smaller and only around $\pm 2-3 \mu \mathrm{A} / \mathrm{cm}^{2}$ (see Figure $3 \mathrm{C}$ ). The reduced current is thus a direct effect of the MBT leaching out of the LDH containers in the coating inhibiting corrosion at the exposed surface.

Figure 4 shows a typical impedance spectrum of an undamaged self-healing coating without corrosion inhibitor and spectra of the same sample with an artificially inflicted defect after 1 and $48 \mathrm{~h}$ immersed in $0.05 \mathrm{M} \mathrm{NaCl}$. The impedance modulus of the unscratched SH sample increases almost linearly with decreasing frequency until $\sim 5 \times 10^{9} \Omega \mathrm{cm}^{2}$ at $0.1 \mathrm{~Hz}$ (Figure 4A). The almost purely capacitive behavior on the whole measured frequency range is reflected in the phase angle of about $-90^{\circ}$ and typical for a barrier coating (Montemor, 2014). This spectrum can be numerically fitted by an equivalent electrical circuit (EEC)
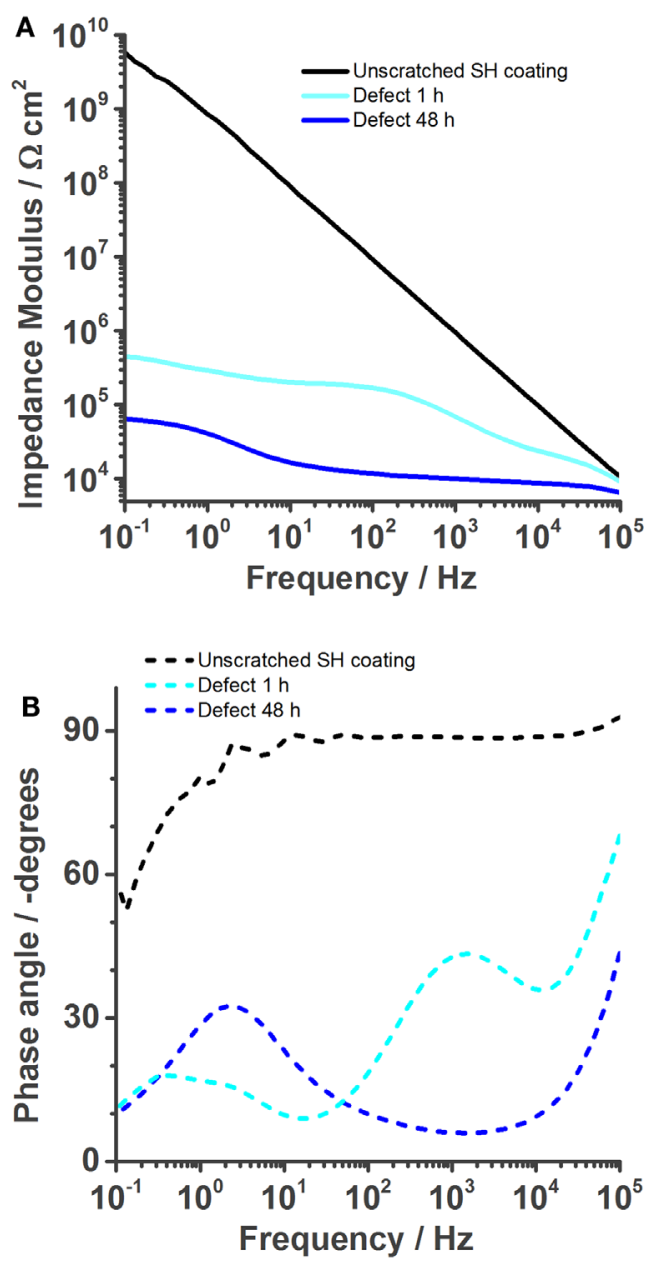

FIGURE 4 | Bode plots of the self-healing reference coating without corrosion inhibitor immersed in $0.05 \mathrm{M} \mathrm{NaCl}$ solution. The undamaged coating shows good barrier properties. (A) But once a defect is inflicted into the coating, its impedance is strongly reduced $(1 \mathrm{~h})$ and further decreases over time (48 h). The phase plot (B) shows the various time constants.

consisting of a capacitor and a resistor in parallel. The capacitor represents the coating capacitance $\left(C_{\text {coat }}\right)$ and the resistor the pore resistance $\left(R_{\text {pore }}\right)$ as commonly described in literature (Walter, 1986; Amirudin and Thieny, 1995; González-García et al., 2007; Bastos et al., 2010; Hauffman et al., 2013). When the barrier is disrupted, the effect of the underlying layers becomes visible. After $1 \mathrm{~h}$ of immersion, the impedance modulus only reaches about $5 \times 10^{5} \Omega \mathrm{cm}^{2}$ at $0.1 \mathrm{~Hz}$ and simultaneously three time constants appear in the respective Bode phase plot (Figure 4B). At the highest frequencies, an artifact of the reference electrode is visible, which contains a high-frequency bypass for the reference electrode and the solution resistance. The first relevant time constant around $1 \mathrm{kHz}$ is related to the coating capacitance and pore resistance. The second one, which is initially less pronounced, shows the effects at the metal-solution interface. A double layer capacitance $\left(C_{\mathrm{dl}}\right)$ and a charge transfer resistance $\left(R_{\mathrm{ct}}\right)$ of the corrosion reaction are commonly used to describe these effects 
of the passivation layer (Walter, 1986; Amirudin and Thieny, 1995; González-García et al., 2007; Bastos et al., 2010; Hauffman et al., 2013). To account for imperfections of the real systems constant phase elements have generally been used instead of pure capacitances. After $48 \mathrm{~h}$, the effect of the coating measured around $10^{3} \mathrm{~Hz}$ disappears in the phase plot (Figure 4B) because of strong coating delamination (see also Figure 7). The time constant at low frequencies can again be described by a double layer capacitance and a charge transfer resistance of the continuing corrosion process. The reduction of $R_{\mathrm{ct}}$ over time has been plot in Figure 5. It decreases from $1 \times 10^{6} \Omega(5 \mathrm{~min}$ after immersion of the sample with a defect in $0.05 \mathrm{M} \mathrm{NaCl}$ ) to about $6 \times 10^{4} \Omega$ after $48 \mathrm{~h}$. As a consequence, also the absolute impedance modulus decreased further to $6 \times 10^{4} \Omega \mathrm{cm}^{2}$ by then.

The results of the EIS measurements of the MASH sample differ from the reference sample; see Figure 6. The barrier properties of the unscratched MASH sample with an impedance modulus of almost $10^{10} \Omega \mathrm{cm}^{2}$ at $0.1 \mathrm{~Hz}$ (Figure 6A) are slightly improved compared with the $\mathrm{SH}$ reference sample. This deviation can be explained by a slightly different coating thickness or a difference in the permittivity (dielectric constant) of the MASH coating containing LDH particles. For numerical fitting, again a single RC circuit is used. One hour after the infliction of a defect, the impedance decreased to $10^{6} \Omega \mathrm{cm}^{2}$. The Bode phase plot (Figure 6B) shows three time constants. At the highest frequencies, the artifact of the reference electrode is still visible. Between 10 and $1000 \mathrm{~Hz}$, the time constant related to the coating capacitance and the pore resistance of the coating is visible. The time constant around $1 \mathrm{~Hz}$ represents again the metal-solution interface with a double layer capacitance and a resistance of the charge transfer. The more pronounced low frequency time constant of the MASH sample indicates a more capacitive behavior originating from the MBT layer formed at the metal surface inhibiting the corrosion reaction. The effect of this layer on the corrosion activity coincides with the results of the SVET measurements earlier in this chapter. Equally, the charge transfer resistance of the MASH sample is higher than

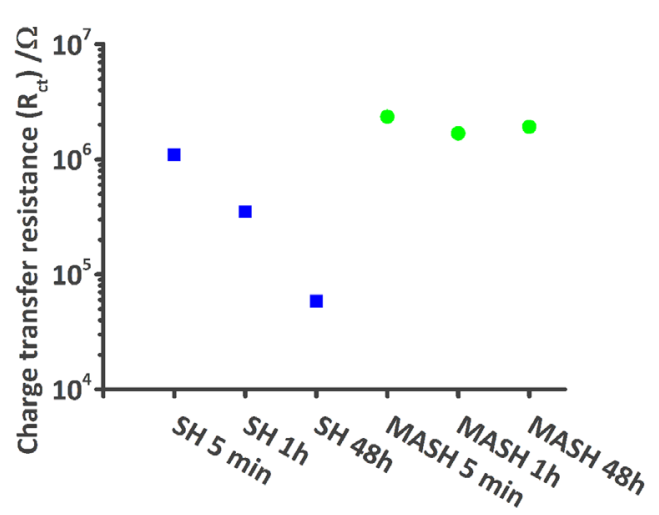

FIGURE 5 | Plot shows the decrease of the modeled charge transfer resistance values of the $\mathrm{SH}$ reference sample (blue squares) during $48 \mathrm{~h}$ in comparison with the more or less constant values of the MASH sample (green dots). the respective values of the self-healing reference sample and stays almost constant (around $2 \times 10^{6} \Omega$ ) during $48 \mathrm{~h}$ as can be seen in Figure 5. This effect is attributed to the MBT inhibiting the corrosion of the metal substrate. The protection of MBT has presumeably even increased over time because the low frequency time constant is more pronounced even though slow coating delamination has started. Furthermore, the disappearance of the time constant at medium frequencies of the MASH sample is slower than its disappearance of the reference coating (it is still visible after $48 \mathrm{~h}$ in Figure 6). This indicates already less severe coating delamination of the MASH sample.

Figure 7 shows microscopy pictures of two samples with a defect after $48 \mathrm{~h}$ of immersion in $0.05 \mathrm{M} \mathrm{NaCl}$ solution. The
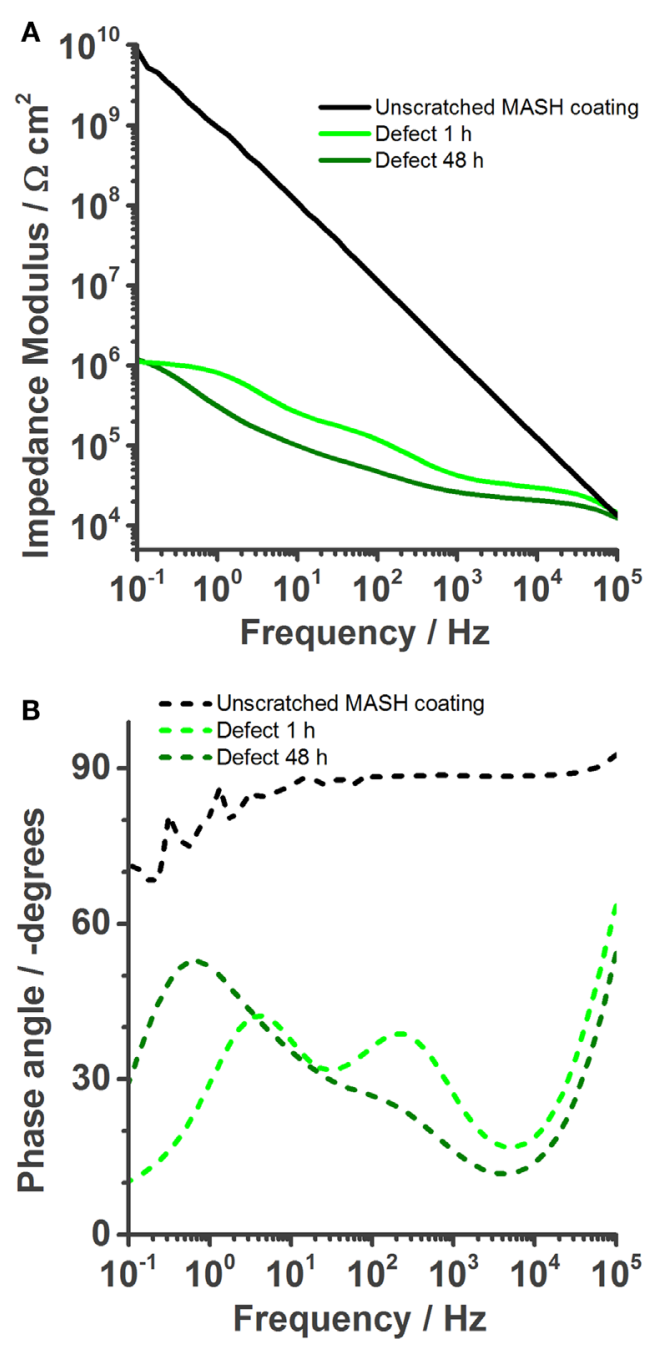

FIGURE 6 | Bode plots of the MASH coating immersed in $0.05 \mathrm{M} \mathrm{NaCl}$ solution. The undamaged coating shows even better barrier properties than the barrier properties of self-healing reference coating. (A) Once a defect is inflicted into the coating, its impedance is naturally also reduced ( $1 \mathrm{~h}$ ). However, over time (48 h) the impedance modulus stays more or less constant. The phase plot (B) shows the reduction of the coating capacitance, but an increase of the phase angle at low frequencies, a result of the protection layer. 


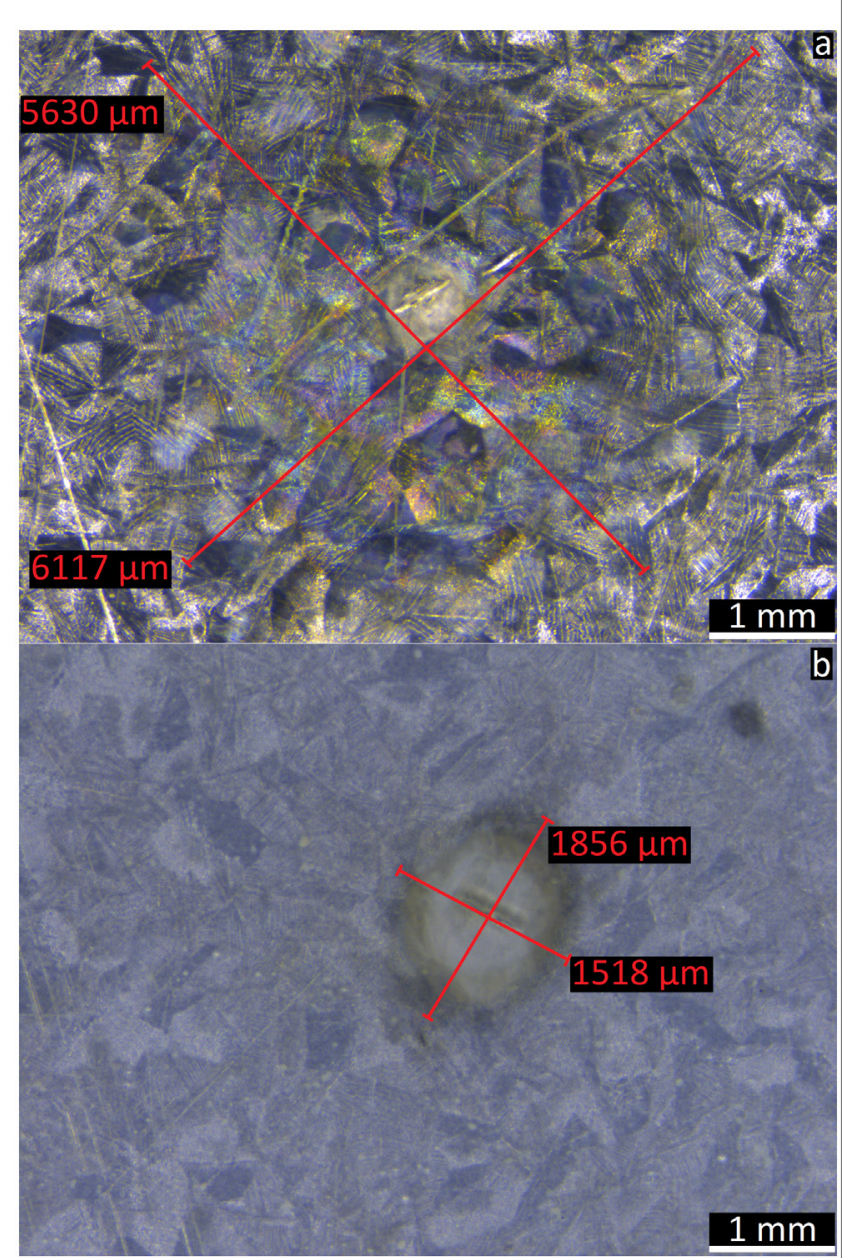

FIGURE 7 | The optical microscope images show the corroded surface underneath the delaminated transparent coating with a defect in the center. The sample without corrosion inhibitor containers (A) shows 13 times bigger delamination than the sample where MBT was released from $\mathrm{LDH}$ in the coating $\mathbf{( B )}$.

coloration of the zinc surface gives a good estimation of the delaminated area. The latter was roughly estimated by measuring the width of the delaminated area twice (red lines). The second measurement was performed perpendicular to the first one. The values were summed up and divided by 2 to calculate the mean diameter. From this value, the delaminated surface was calculated using the formula for the surface of a disk $\left[A=(d / 2)^{2} \times \pi\right]$. The MASH sample has a delaminated surface of $2 \mathrm{~mm}^{2}$, and the coating without inhibitor loaded containers has a delaminated surface of $27 \mathrm{~mm}^{2}$. Thus, the addition of MLDH to the reference coating reduces the delaminated area around a defect after $48 \mathrm{~h}$ in immersion by a factor 13 .

Next, the delaminated area underneath the coating was investigated for the presence of corrosion products and corrosion inhibitor. Therefore, the coating of the samples shown in Figure 7B was removed and the metal surface was analyzed by SEM-EDX and Raman spectroscopy. At the delamination front, three different areas could be distinguished (Figure 8). On the left side marked with the number 1 is the surface where the coating was not yet delaminated. It consists of coating traces, zinc, oxygen, and about $1 \%$ of aluminum. The area in the middle (2) is the delamination front. Small amounts of sulfur were detected by EDX. The rest of the delaminated area looks like area 3 on the right side of Figure 8. Here, again sulfur and additionally 2 at $\%$ of chlorine were detected on the surface by EDX, the latter being the main responsible for the corrosive attack of the zinc surface and coating delamination. The detected sulfur originates from MBT, which was shown by another series of Raman spectroscopy measurements (Figure 9). The spectrum of the MBT solution on the SERS probe was again taken as a reference. The uncorroded HDG surface far away from the defect is Raman inactive and basically just shows background noise in its spectrum (blue). The optical image on the bottom shows the uncorroded surface where the spectrum was recorded. Around the defect where the coating was already delaminated as a consequence of the corrosive attack, the spectrum contains weak MBT peaks (orange). The microscope image in the middle shows the respective surface. The same peaks - but more intense - are present in the spectrum taken just next to the defect (red), with the optical image of the side of the defect in the top right corner. Because MBT is present at the metal surface where corrosive attack by chloride ions took place and it was shown to be automatically released from $\mathrm{LDH}$ in the presence of aggressive ions (Poznyak et al., 2009), we conclude that MBT is responsible for the corrosion inhibition at the HDG surface.

\section{Second Self-Healing Action: Effect of the Shape-Recovery Coating}

When the coating was foreseen with a scratch or a defect, the energy that was put into the coating by mechanically deforming it, was stored by the material. However, it can be released by heating the sample. Surpassing the glass transition temperature of PCL, increases the mobility of the molecular network structure of the coating so much that it can recover its original shape with that stored energy, resulting in the closure of the defect. Thereby, no chemical bonds (other than by the creation of the defect) are broken or formed in this process, and thus theoretically it is infinitely repeatable (Westbrook et al., 2011; Lutz et al., 2015).

A similar defect as shown for the ones before was inflicted to the coating of another MASH sample. The sample was immersed in $0.05 \mathrm{M} \mathrm{NaCl}$ solution and its impedance was measured once (Figure 10). Next, after a total of $<20 \mathrm{~min}$, this sample was taken out of the solution, wiped dry with a tissue, and heated to $60^{\circ} \mathrm{C}$ for $2 \mathrm{~min}$ to trigger the non-autonomous on-demand healing action. This procedure was previously shown to be sufficient to heal defects in the coating without MLDH particles (Lutz et al., 2015). The performance of the healed sample was again analyzed with EIS. Figure 10 shows the spectra of the undamaged sample, the sample with a defect, the sample that was heated to $60^{\circ} \mathrm{C}$ for $2 \mathrm{~min}$. The undamaged and the damaged MASH samples show similar spectra as described before in Figure 6. The sample after healing has clearly increased barrier properties which we relate to the closed defect. The impedance at $0.1 \mathrm{~Hz}$ is $10^{8} \Omega \mathrm{cm}^{2}$.

Numerical fitting of the spectra with either one (undamaged and healed sample) or three RC circuits (damaged sample) gives 


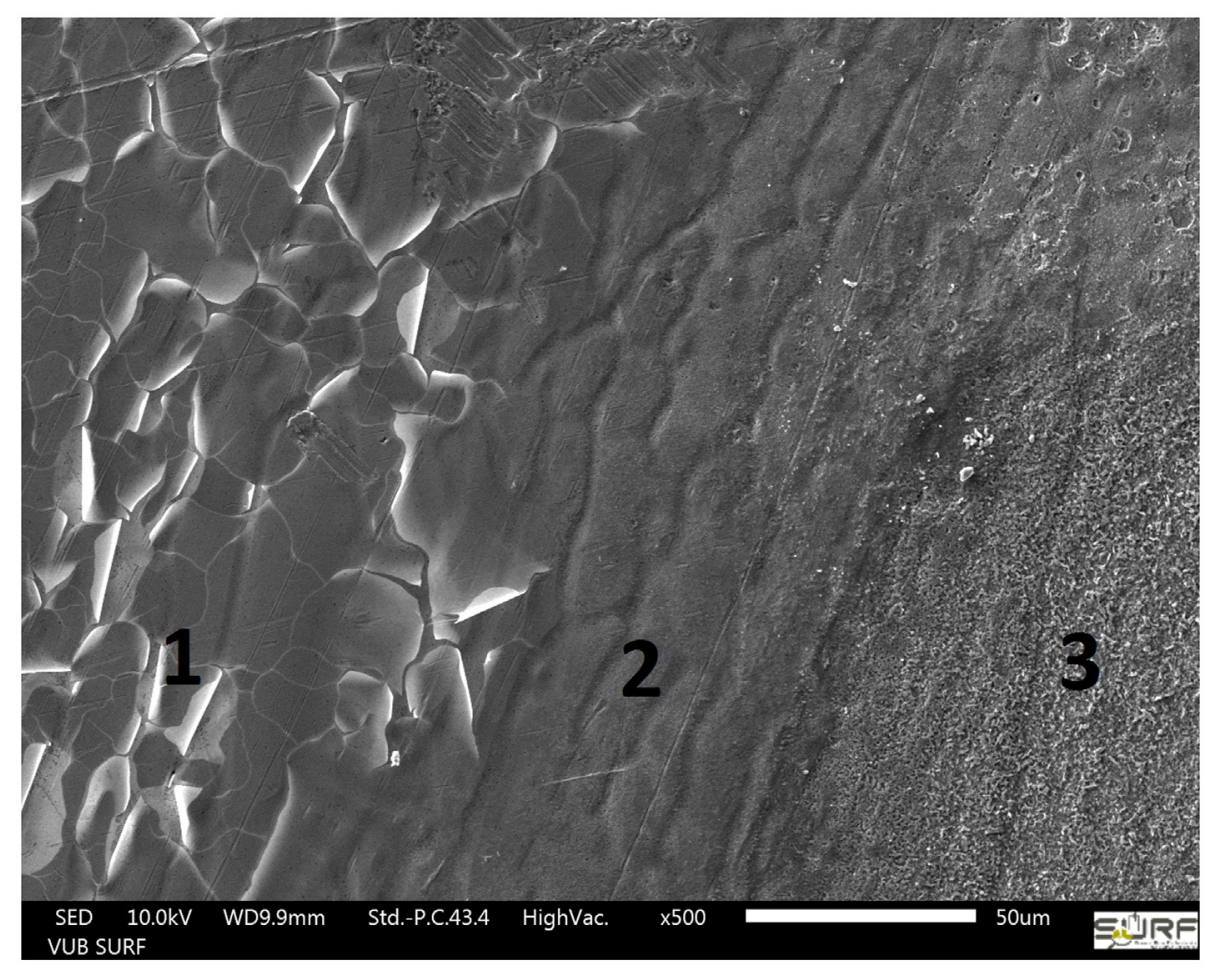

FIGURE 8 | SEM image of the HDG surface at the delamination front (2): left side (1) not attacked area, right side (3) corroded surface.

the respective values for $C_{\text {coat }}$ and $R_{\text {pore. }}$ The capacitances, although denoted, for example, as $C_{\text {coat }}$, were again fitted with CPE's instead of pure capacitances. Figure 11 shows that $C_{\text {coat }}$ increases when a defect is made into the coating and $R_{\text {pore }}$ decreases at the same time. However, by heating the MASH sample, thereby closing the defect, $C_{\text {coat }}$ decreases back to almost the pristine values just above $10^{-10}$. $R_{\text {pore }}$ also increases, but because the interface between the two sides of the scratch still persists, does not fully recover $\left(2 \times 10^{8}\right.$ vs. $\left.10^{10} \Omega\right)$.

If the defect was fully healed, we would expect all values to be similar to the one of the undamaged MASH sample. However, the values of the healed sample are between the one of the pristine sample and the one of the damaged one. The barrier properties of the coating could thus be increased by heating the sample providing better corrosion protection but could not be fully restored. The two phase boundaries came closer to each other but are still visible under the optical microscope (see Figure S1 in Supplementary Material).

Also, the OCP provides an indication for the partial recovery, see Figure 12. The undamaged samples ( $\mathrm{SH}$ reference and $\mathrm{MASH}$ ) have an OCP of $-0.62 \mathrm{~V}$ and $-0.56 \mathrm{~V}$, respectively. With a damage the OCP decreases to $-0.95 \mathrm{~V}$ for both samples as the HDG surface is determining the corrosion potential. After healing, the potential of the $\mathrm{SH}$ reference sample rose again to $-0.77 \mathrm{~V}$ and the potential of the MASH sample to $-0.67 \mathrm{~V}$. The difference in potential between the undamaged and healed sample is due to the non-perfect healing of the damage. Therefore, it is not surprising that the impedance did not recover to the same level as prior to the infliction of the defect.

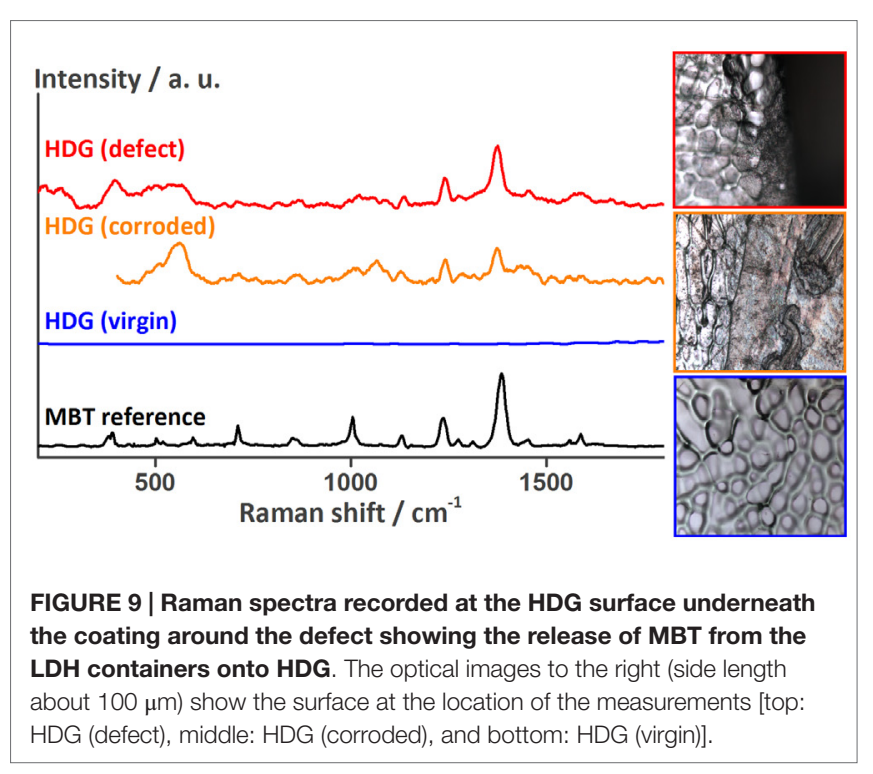

However, the effect of the defect closing is much higher than the one of the MBT leached from the $\mathrm{LDH}$ particles.

\section{CONCLUSION}

We demonstrate for the first time that the combination of two different types of self-healing mechanisms, namely, an intrinsic 

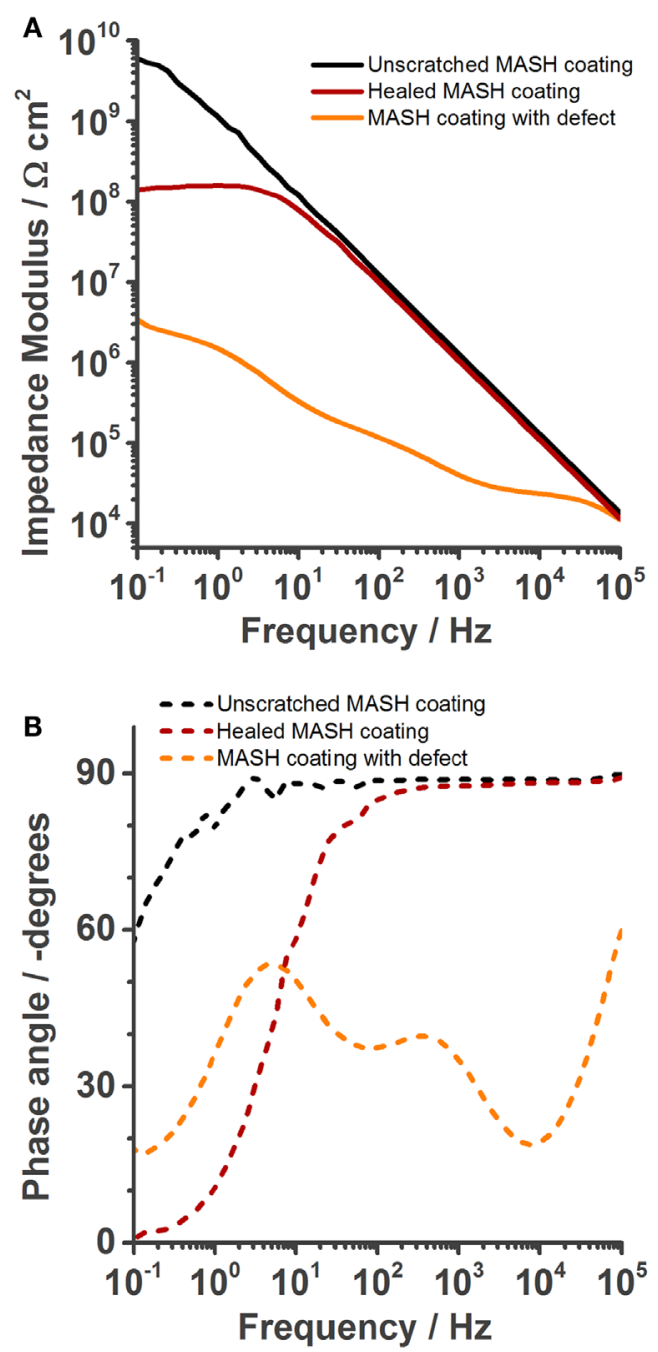

FIGURE 10 | Electrochemical impedance spectroscopy spectra recorded in $0.05 \mathrm{M} \mathrm{NaCl}$ solution after OCP was constant (about $5 \mathrm{~min}$ ), showing the increase of barrier properties achieved by healing of the self-healing polymer at $60^{\circ} \mathrm{C}$ for $2 \mathrm{~min}$. (A) Bode modulus, (B) bode phase plot.

action and an extrinsic action in a single coating. Smart corrosion inhibitor containers embedded in the shape-recovery coating automatically release 2-MBT to inhibit corrosion of a HDG steel sample with a defect in the self-healing polymer coating. Alternatively, the shape-recovery polymer coating closes the defect on-demand when heating the sample for $2 \mathrm{~min}$ above $60^{\circ} \mathrm{C}$ preventing ingress of solution, and thus corrosive attack. By combining the two self-healing mechanisms deficiencies of the single self-healing systems can be overcome. In particular, this leads to the following conclusions:

- The barrier properties of both undamaged coatings are similar, with slightly higher impedance of the MASH sample presumably caused by the finely distributed LDH containers in the coating.

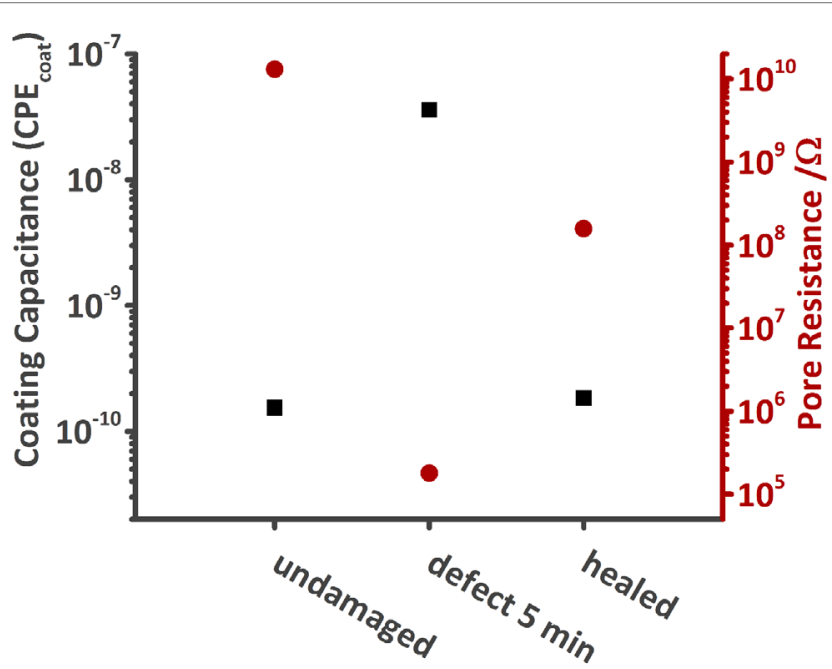

FIGURE 11 | Comparison of the coating capacitance and $\boldsymbol{R}_{\text {pore }}$ of the undamaged, damaged, and healed MASH sample immersed in 0.05 M $\mathrm{NaCl}$ solution. Both values recover after heating the $\mathrm{MASH}$ sample to $60^{\circ} \mathrm{C}$.

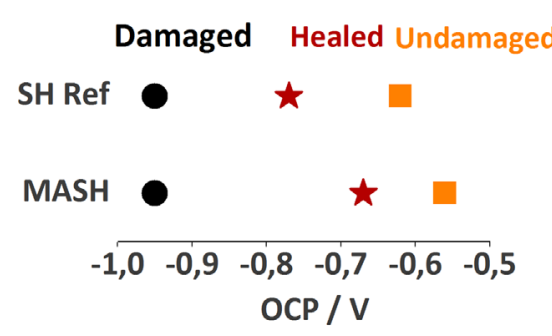

FIGURE 12 | The different open circuit potentials of the samples immersed in $0.05 \mathrm{M} \mathrm{NaCl}$ after equilibration against a $\mathbf{A g} \mid \mathbf{A g C l}$ reference electrode.

- Upon immersion of a sample with a defect in $0.05 \mathrm{M}$ sodium chloride solution, the additional protection of MBT leached out of the LDH containers can be observed with EIS and the presence of MBT at the protected metal surface can be verified by SEM-EDX and Raman spectroscopy.

- SVET measurements show a clear decrease of the corrosion activity of the MASH sample with a defect and when the defect is healed.

- After 2 days of immersion in this solution, the delaminated area underneath the coating of the MASH sample is significantly smaller than the one of the pure shape-recovery coating.

- Thermally induced on-demand self-healing partly closes the defect within 2 min by shape recovery of the polymeric coating.

However, for better long-time corrosion protection and reduced delamination adhesion promoters certainly need to be added to the coating system. 


\section{ACKNOWLEDGMENTS}

AL wants to thank Kitty Baert for her support and advice with the Raman Spectroscope.

\section{FUNDING}

The authors wish to acknowledge Strategic Initiative Materials (SIM) and Agentschap voor Innovatie door Wetenschap en

\section{REFERENCES}

Amirudin, A., and Thieny, D. (1995). Application of electrochemical impedance spectroscopy to study the degradation of polymer-coated metals. Prog. Org. Coat. 26, 1-28. doi:10.1016/0300-9440(95)00581-1

Andreeva, D. V., and Shchukin, D. G. (2008). Smart self-repairing protective coatings. Mater. Today 11, 24-30.

Bastos, A. C., Zheludkevich, M. L., and Ferreira, M. G. S. (2008). A SVET investigation on the modification of zinc dust reactivity. Prog. Org. Coat. 63, 282-290. doi:10.1016/j.porgcoat.2008.01.013

Bastos, A. C., Zheludkevich, M. L., Klüppel, I., Grundmeier, G., and Ferreira, M. G. S. (2010). Modification of zinc powder to improve the corrosion resistance of weldable primers. Prog. Org. Coat. 69, 184-192. doi:10.1016/j. porgcoat.2010.04.021

Blaiszik, B. J., Kramer, S. L. B., Olugebefola, S. C., Moore, J. S., Sottos, N. R., and White, S. R. (2010). Self-healing polymers and composites. Annu. Rev. Mater. Res. 40, 179-211. doi:10.1146/annurev-matsci-070909-104532

Borisova, D., Möhwald, H., and Shchukin, D. G. (2013a). Influence of embedded nanocontainers on the efficiency of active anticorrosive coatings for aluminum alloys part II: influence of nanocontainer position. ACS Appl. Mater. Interfaces 5, 80-87. doi:10.1021/am302141y

Borisova, D., Akçakayıran, D., Schenderlein, M., Möhwald, H., and Shchukin, D. G. (2013b). Nanocontainer-based anticorrosive coatings: effect of the container size on the self-healing performance. Adv. Funct. Mater. 23, 3799-3812. doi:10.1002/adfm.201203715

Brancart, J., Scheltjens, G., Muselle, T., Van Mele, B., Terryn, H., and Van Assche, G. (2012). Atomic force microscopy-based study of self-healing coatings based on reversible polymer network systems. J. Intell. Mater. Syst. Struct. 25, 40-46. doi:10.1177/1045389X12457100

Brown, E. N., Kessler, M. R., Sottos, N. R., and White, S. R. (2003). In-situ poly(urea-formaldehyde) microencapsulation of dicyclopentadiene. J. Microencapsul. 20, 719-730. doi:10.1080/0265204031000154160

D'Hollander, S., Van Assche, G., Van Mele, B., and Du Prez, F. (2009). Novel synthetic strategy toward shape memory polyurethanes with a well-defined switching temperature. Polymer 50, 4447-4454. doi:10.1016/j. polymer.2009.07.021

Döhler, D., Peterlik, H., and Binder, W. H. (2015). A dual crosslinked self-healing system: supramolecular and covalent network formation of four-arm star polymers. Polymer 69, 264-273. doi:10.1016/j.polymer.2015.01.073

Fedrizzi, L. (2011). Self-Healing Properties of New Surface Treatments. Leeds: EFC.

Ferrer, E. L., Rollon, A. P., Mendoza, H. D., Lafont, U., and Garcia, S. J. (2014). Doubledoped zeolites for corrosion protection of aluminium alloys. Microporous Mesoporous Mater. 188, 8-15. doi:10.1016/j.micromeso.2014.01.004

García, S. J., Fischer, H. R., and van der Zwaag, S. (2011). A critical appraisal of the potential of self healing polymeric coatings. Prog. Org. Coat. 72, 211-221. doi:10.1016/j.porgcoat.2011.06.016

González-García, Y., González, S., and Souto, R. M. (2007). Electrochemical and structural properties of a polyurethane coating on steel substrates for corrosion protection. Corros. Sci. 49, 3514-3526. doi:10.1016/j.corsci.2007.03.018

González-García, Y., Mol, J. M. C., Muselle, T., De Graeve, I., Van Assche, G., Scheltjens, G., et al. (2011a). A combined mechanical, microscopic and local electrochemical evaluation of self-healing properties of shape-memory polyurethane coatings. Electrochim. Acta 56, 9619-9626. doi:10.1016/j. electacta.2011.03.081

González-García, Y., Mol, J. M. C., Muselle, T., De Graeve, I., Van Assche, G., Scheltjens, G., et al. (2011b). SECM study of defect repair in self-healing
Technologie (IWT) for funding the Novel Active Protection Systems on Metals (NAPROM) project and the Selfhealing - repairing coatings (SHREC) project as part of the SHE program on Self-Healing Engineered materials.

\section{SUPPLEMENTARY MATERIAL}

The Supplementary Material for this article can be found online at http://journal.frontiersin.org/article/10.3389/fmats.2015.00073

polymer coatings on metals. Electrochem. commun. 13, 169-173. doi:10.1016/j. elecom.2010.12.005

Grigoriev, D. O., Köhler, K., Skorb, E., Shchukin, D. G., and Möhwald, H. (2009). Polyelectrolyte complexes as a "smart" depot for self-healing anticorrosion coatings. Soft Matter 5, 1426. doi:10.1039/b815147d

Hansen, C. J., Wu, W., Toohey, K. S., Sottos, N. R., White, S. R., and Lewis, J. A. (2009). Self-healing materials with interpenetrating microvascular networks. Adv. Mater. 21, 4143-4147. doi:10.1002/adma.200900588

Hauffman, T., Van Ingelgem, Y., Breugelmans, T., Tourwé, E., Terryn, H., and Hubin, A. (2013). Dynamic, in situ study of self-assembling organic phosphonic acid monolayers from ethanolic solutions on aluminium oxides by means of odd random phase multisine electrochemical impedance spectroscopy. Electrochim. Acta 106, 342-350. doi:10.1016/j.electacta.2013.04.025

He, J., Wei, M., Li, B., Kang, Y., Evans, D. G., and Duan, X. (2006). "Preparation of layered double hydroxides," in Layered Double Hydroxides, Vol. 119. SpringerVerlag: Berlin/Heidelberg, 89-119.

Hillewaere, X. K. D., and Du Prez, F. E. (2015). Fifteen chemistries for autonomous external self-healing polymers and composites. Prog. Polym. Sci. 49-50, 121-153. doi:10.1016/j.progpolymsci.2015.04.004

Hong, Y., Devarapalli, V. K., Roy, D., and Babu, S. V. (2007). Synergistic roles of dodecyl sulfate and benzotriazole in enhancing the efficiency of CMP of copper. J. Electrochem. Soc. 154, H444. doi:10.1149/1.2717410

Jorcin, J.-B., Scheltjens, G., Van Ingelgem, Y., Tourwé, E., Van Assche, G., De Graeve, I., et al. (2010). Investigation of the self-healing properties of shape memory polyurethane coatings with the "odd random phase multisine" electrochemical impedance spectroscopy. Electrochim. Acta 55, 6195-6203. doi:10.1016/j.electacta.2010.01.027

Kartsonakis, I. A., Athanasopoulou, E., Snihirova, D., Martins, B., Koklioti, M. A., Montemor, M. F., et al. (2014). Multifunctional epoxy coatings combining a mixture of traps and inhibitor loaded nanocontainers for corrosion protection of AA2024-T3. Corros. Sci. 85, 147-159. doi:10.1016/j.corsci.2014.04.009

Kukkadapu, R. K., Witkowski, M. S., and Amonette, J. E. (1997). Synthesis of a low-carbonate high-charge hydrotalcite-like compound at ambient pressure and atmosphere. Chem. Mater. 9, 417-419. doi:10.1021/cm960536c

Luo, X., and Mather, P. T. (2013). Shape memory assisted self-healing coating. ACS Macro Lett. 2, 152-156. doi:10.1021/mz400017x

Lutz, A., van den Berg, O., Van Damme, J., Verheyen, K., Bauters, E., De Graeve, I., et al. (2015). A shape recovery polymer coating for the corrosion protection of metallic surfaces. ACS Appl. Mater. Interfaces 7, 175-183. doi:10.1021/ am505621x

Maia, F., Tedim, J., Bastos, A. C., Ferreira, M. G. S., and Zheludkevich, M. L. (2013). Nanocontainer-based corrosion sensing coating. Nanotechnology 24, 415502. doi:10.1088/0957-4484/24/41/415502

Maia, F., Tedim, J., Lisenkov, A. D., Salak, A. N., Zheludkevich, M. L., Maia, F., et al. (2012). Silica nanocontainers for active corrosion protection. Nanoscale 4, 1287-1298. doi:10.1039/c2nr11536k

Markley, T. A., Hughes, A. E., Ang, T. C., Deacon, G. B., Junk, P., and Forsyth, M. (2007). Influence of praseodymium. Electrochem. Solid State Lett. 10, C72. doi:10.1149/1.2790724

Montemor, M., Trabelsi, W., Lamaka, S., Yasakau, K., Zheludkevich, M., Bastos, A., et al. (2008). The synergistic combination of bis-silane and $\mathrm{CeO} 2 \cdot \mathrm{ZrO} 2$ nanoparticles on the electrochemical behaviour of galvanised steel in $\mathrm{NaCl}$ solutions. Electrochim. Acta 53, 5913-5922. doi:10.1016/j.electacta.2008.03.069

Montemor, M. F. (2014). Functional and smart coatings for corrosion protection: a review of recent advances. Surf. Coat. Technol. 258, 17-37. doi:10.1016/j. surfcoat.2014.06.031 
Montemor, M. F., Snihirova, D. V., Taryba, M. G., Lamaka, S. V., Kartsonakis, I. A., Balaskas, A. C., et al. (2012). Evaluation of self-healing ability in protective coatings modified with combinations of layered double hydroxides and cerium molibdate nanocontainers filled with corrosion inhibitors. Electrochim. Acta 60, 31-40. doi:10.1016/j.electacta.2011.10.078

Müller, B., and Imblo, G. (1996). Heterocycles as corrosion inhibitors for zinc pigments in aqueous alkaline media. Corros. Sci. 38, 293-300. doi:10.1016/0010-938X(96)00122-9

Poznyak, S. K., Tedim, J., Rodrigues, L. M., Salak, A. N., Zheludkevich, M. L., Dick, L. F. P., et al. (2009). Hydroxides intercalated with guest organic inhibitors for anticorrosion applications. ACS Appl. Mater. Interfaces 1, 2353-2362. doi:10.1021/am900495r

Rodriguez, E. D., Luo, X., and Mather, P. T. (2011). Linear/network poly( $\varepsilon$-caprolactone) blends exhibiting shape memory assisted self-healing (SMASH). ACS Appl. Mater. Interfaces 3, 152-161. doi:10.1021/am101012c

Samadzadeh, M., Boura, S. H., Peikari, M., Kasiriha, S. M., and Ashrafi, A. (2010). A review on self-healing coatings based on micro/nanocapsules. Prog. Org. Coat. 68, 159-164. doi:10.1016/j.porgcoat.2010.01.006

Scheltjens, G., Diaz, M. M., Brancart, J., Van Assche, G., and Van Mele, B. (2013). A self-healing polymer network based on reversible covalent bonding. React. Funct. Polym. 73, 413-420. doi:10.1016/j.reactfunctpolym.2012.06.017

Serdechnova, M., Kallip, S., Ferreira, M. G. S., and Zheludkevich, M. L. (2014). Active self-healing coating for galvanically coupled multi-material assemblies. Electrochem. commun. 41, 51-54. doi:10.1016/j.elecom.2014.01.023

Shchukin, D. G., Grigoriev, D. O., and Möhwald, H. (2010). Application of smart organic nanocontainers in feedback active coatings $\uparrow$. Soft Matter 6, 720-725. doi:10.1039/b918437f

Toohey, K. S., Sottos, N. R., Lewis, J. A., Moore, J. S., and White, S. R. (2007). Self-healing materials with microvascular networks. Nat. Mater. 6, 581-585. doi:10.1038/nmat 1934

Walter, G. W. (1986). A review of impedance plot methods used for corrosion performance analysis of painted metals. Corros. Sci. 26, 681-703. doi:10.1016/0010-938X(86)90033-8

Westbrook, K. K., Mather, P. T., Parakh, V., Dunn, M. L., Ge, Q., Lee, B. M., et al. (2011). Two-way reversible shape memory effects in a free-standing polymer composite. Smart Mater. Struct. 20, 065010. doi:10.1088/0964-1726/20/6/065010

White, S. R., Sottos, N. R., Geubelle, P. H., Moore, J. S., Kessler, M. R., Sriram, S. R., et al. (2001). Autonomic healing of polymer composites. Nature 409, 794-797. doi:10.1038/35057232

Wouters, M., Craenmehr, E., Tempelaars, K., Fischer, H., Stroeks, N., and van Zanten, J. (2009). Preparation and properties of a novel remendable coating concept. Prog. Org. Coat. 64, 156-162. doi:10.1016/ j.porgcoat.2008.09.023

Wu, D., Meure, S., and Solomon, D. (2008). Self-healing polymeric materials: a review of recent developments. Prog. Polym. Sci. 33, 479-522. doi:10.1016/j. progpolymsci.2008.02.001

Yang,H.,Sun, Y., Ji,J.,Song, W.,Zhu,X., Yao, Y., etal.(2008).2-Mercaptobenzothiazole monolayers on zinc and silver surfaces for anticorrosion. Corros. Sci. 50, 3160-3167. doi:10.1016/j.corsci.2008.08.036

Yasakau, K. A., Tedim, J., Zheludkevich, M. L., Drumm, R., Shem, M., Wittmar, M., et al. (2012). Cerium molybdate nanowires for active corrosion protection of aluminium alloys. Corros. Sci. 58, 41-51. doi:10.1016/j. corsci.2012.01.012

Yuan, Y. C. (2008). Self healing in polymers and polymer composites. Concepts, realization and outlook: a review. Express Polym. Lett. 2, 238-250. doi:10.3144/ expresspolymlett.2008.29

Zadeh, M. A., Garcia, S. J. J., van der Zwaag, S., Abdolah Zadeh, M., van der Zwaag, S., and Garcia, S. J. J. (2013). Routes to extrinsic and intrinsic self-healing corrosion protective sol-gel coatings: a review. Self Healing Mater. 1, 1-18. doi:10.2478/shm-2013-0001

Zheludkevich, M. L., Poznyak, S. K., Rodrigues, L. M., Raps, D., Hack, T., Dick, L. F., et al. (2010). Active protection coatings with layered double hydroxide nanocontainers of corrosion inhibitor. Corros. Sci. 52, 602-611. doi:10.1016/j. corsci.2009.10.020

Zheludkevich, M. L., Tedim, J., and Ferreira, M. G. S. (2012). “ Smart “ coatings for active corrosion protection based on multi-functional micro and nanocontainers. Electrochim. Acta 82, 314-323. doi:10.1016/j.electacta.2012.04.095

Zwaag, S. (2007). Self Healing Materials. Springer: Dordrecht.

Conflict of Interest Statement: The authors declare that the research was conducted in the absence of any commercial or financial relationships that could be construed as a potential conflict of interest.

The reviewer Wolfram Fürbeth and the handling editor Mikhail Zheludkevich declared a current collaboration and the handling editor states that the process nevertheless met the standards of a fair and objective review.

Copyright (c) 2016 Lutz, van den Berg, Wielant, De Graeve and Terryn. This is an open-access article distributed under the terms of the Creative Commons Attribution License (CC BY). The use, distribution or reproduction in other forums is permitted, provided the original author(s) or licensor are credited and that the original publication in this journal is cited, in accordance with accepted academic practice. No use, distribution or reproduction is permitted which does not comply with these terms. 